\title{
Stability of longitudinal coupling for Josephson charge qubits
}

\author{
Carsten Hutter, ${ }^{1,2}$ Yuriy Makhlin, ${ }^{3}$ Alexander Shnirman, ${ }^{1}$ and Gerd Schön ${ }^{1}$ \\ ${ }^{1}$ Institut für Theoretische Festkörperphysik and DFG-Center for Functional Nanostructures (CFN), \\ Universität Karlsruhe, D-76128 Karlsruhe, Germany \\ ${ }^{2}$ Department of Physics, Stockholm University, AlbaNova University Center, SE-106 91 Stockholm, Sweden \\ ${ }^{3}$ Landau Institute for Theoretical Physics, Kosygin st. 2, 119334 Moscow, Russia
}

\begin{abstract}
For inductively coupled superconducting quantum bits, we determine the conditions when the coupling commutes with the single-qubit terms. We show that in certain parameter regimes such longitudinal coupling can be stabilized with respect to variations of the circuit parameters. In addition, we analyze its stability against fluctuations of the control fields.
\end{abstract}

PACS numbers: 85.25.Hv, 03.67.Lx, 74.81.Fa, 85.25.Cp

Among potential realizations of scalable quantum computers 1 , superconducting qubits belong to the promising candidates 2.3 .4 . Experimentally, coupling between quantum bits has been demonstrated $5,6,7,8,9$, as well as coupling of a qubit to an oscillator ${ }^{10.11}$. So far most experiments have been performed with fixed coupling, but for flexible and precise coherent control of a larger number of qubits, a tunable coupling is preferable. First demonstrations of such tunable coupling have been provided only recently $\underline{12,13}$.

Although it is not necessary, for convenience and precision of the operations, one wishes to be able to switch single-qubit and coupling terms on and off independently. On the other hand, in many physical realizations of qubits, coupling terms and single-qubit terms are switched by the same control parameters. Especially in designs which couple qubits via an LC-oscillator ${ }^{14.15}$, the coupling terms are present only when simultaneously single-qubit terms are turned on. If this cannot be avoided, one should at least make efforts to ensure that the coupling and single-qubit terms commute with each other, a situation which we denote as "longitudinal" coupling. In this case, the time-evolution operator factorizes into parts due to the coupling and due to singlequbit terms. If the coupling can be switched off, one can easily undo unwanted single-qubit operations and thus produce a "pure" two-qubit gate. Moreover, if one can control the longitudinal-coupling strength, the resulting operation is sensitive only to the time integral and not to the detailed profile of the control pulse, a property which makes operation easier and more stable.

Here, we investigate tunable longitudinal-coupling schemes for Josephson charge qubits. At the charge degeneracy point, where dephasing is weakest, capacitive (charge-charge) coupling is always "transverse" (i.e. $\sigma_{z} \cdot \sigma_{z}$ coupling, while the single qubit term is proportional to $\sigma_{x}$ ). Also the tunable inductive (currentcurrent) coupling scheme proposed in Refs. 2, 14, where the qubits are coupled to a common inductor, is purely transverse. However, this scheme can be modified in such a way that the coupling at the symmetry point becomes purely longitudinal $15,16,17$.

The longitudinal-coupling designs proposed in Refs. 15 ,
16, 17 assume identical junctions within each qubit. As we will show below, variations of the junction parameters (especially critical currents and capacitances), which are unavoidable due to fabrication errors, add transverse terms to the coupling, which can not be tuned to zero. This problem has also been noted in Ref. 18. Here, we propose and analyze a design with longitudinal inductive coupling, which is stable against such imperfections, in the sense that by tuning control parameters, we can reach a point with purely longitudinal coupling.

The simplest method to compensate for variations in the critical currents of the junctions in the circuit is to replace them by dc-SQUIDs or more complicated circuits, which allow tuning of the effective Josephson couplings via applied, individually controlled magnetic fluxes. However, in order to reach effectively identical junctions, one also needs tunable capacitances, for which there is no simple and efficient solution. Nevertheless, we found a design where, in spite of the spread in fabrication parameters, it is possible to achieve longitudinal coupling merely by tuning fluxes to specific values. For designs with two junctions per qubit (those of Refs. 15, 16, 17 can be viewed as such), this is possible even when the Josephson energies of these junctions are different, provided that, in addition, the capacitances are different. In other words, the system should deliberately be fabricated asymmetric.

Further, we analyze the stability with respect to timedependent fluctuations of the magnetic fluxes. Within the region of parameters, where tuning to purely longitudinal coupling is possible, the instability against fluctuations of fluxes increases with increasing asymmetry of the capacitances. Thus, on one hand, the system should be fabricated asymmetric in order to allow tuning to longitudinal coupling; on the other hand, in order to minimize the instability with respect to fluctuations in the applied fluxes, one should keep the values of the capacitances as close to each other as the first requirement permits.

The system. As a specific example, we consider inductively coupled charge qubits as shown in Fig. 1 For particular values of the Josephson energies $E_{2}^{j}$ and capacitances $C_{a}^{j}$, it reduces to the design of Refs. 2. 14 with transverse coupling or to that of Ref. 15 with longitudi- 
nal coupling. The coupling can be controlled by fluxes applied in the loops. We use an upper index to enumerate qubits and a lower index a/b to distinguish junctions above and below the island of each qubit.

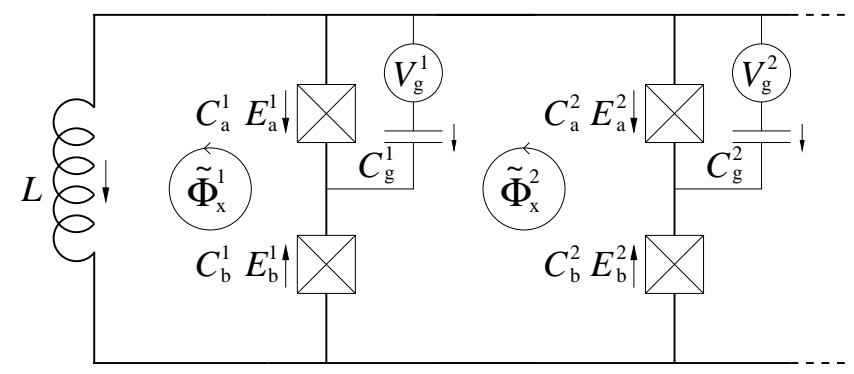

FIG. 1: Charge qubits coupled to an $L C$-circuit. Earlier proposals with either longitudinal ${ }^{15}$ or transverse ${ }^{2,14}$ coupling can be considered as particular cases of this design. The fluxes shown here are applied in the loops between the qubits, such that the total flux bias between the inductance and a qubit $j$ is given as $\Phi_{\mathrm{x}}^{j}=\sum_{k=1}^{j} \tilde{\Phi}_{\mathrm{x}}^{k}$. The junctions may be replaced by SQUID loops to achieve tunable Josephson energies.

If $N$ qubits are coupled to the inductor $L$, there are $N+1$ independent phase variables in the system. As one of them, we choose the phase drop $\phi_{L}=2 \pi \Phi_{L} / \Phi_{0}$, related to the flux $\Phi_{L}$ through the inductor. Furthermore, for each qubit $j$, we introduce the phase $\phi^{j}$ across the junction above the qubit island. The system is then described by the Hamiltonian

$$
\begin{aligned}
& H=\sum_{j=1}^{N} \frac{\left(2 e n^{j}-q^{j}\right)^{2}}{2 C_{\Sigma}^{j}}+\frac{1}{2 C_{s}}\left[Q+\sum_{j=1}^{N} k_{\mathrm{a}}^{j}\left(2 e n^{j}-q^{j}\right)\right]^{2} \\
& +\frac{\Phi_{L}^{2}}{2 L}-\sum_{j=1}^{N} E_{\mathrm{a}}^{j} \cos \phi^{j}-\sum_{j=1}^{N} E_{\mathrm{b}}^{j} \cos \left(\phi^{j}-\phi_{L}-\phi_{\mathrm{x}}^{j}\right)
\end{aligned}
$$

Here $n^{j}$ is the number of excess Cooper-pair charges on the island of qubit $j$, canonically conjugate to $\phi^{j}$. The conjugate variable to $\Phi_{L}$ is the charge $Q$ on the capacitors of the lower junctions of all qubits. The gate charges of the qubit are defined as $q^{j}=C_{\mathrm{g}}^{j} V_{\mathrm{g}}^{j}$, where $V_{\mathrm{g}}^{j}$ is the gate voltage and $C_{\mathrm{g}}^{j}$ is the corresponding capacitance. For simplicity, we consider constant applied fluxes. The total qubit charge, screened by capacitances with ratios $k_{\mathrm{a}}^{j} \equiv C_{\mathrm{b}}^{j} / C_{\Sigma}^{j}$, acts as gate charge of the oscillator. The total capacitance of each island is $C_{\Sigma}^{j}=C_{\mathrm{a}}^{j}+C_{\mathrm{b}}^{j}+C_{\mathrm{g}}^{j}$. The Josephson coupling terms of the Hamiltonian contain the total phase bias between each qubit and the inductor, $\phi_{\mathrm{x}}^{j}=\sum_{k=1}^{j} \frac{2 \pi}{\Phi_{0}} \tilde{\Phi}_{\mathrm{x}}^{j}$. We further introduced the total capacitance of the $L C$-circuit, $C_{s}=\sum_{j=1}^{N}\left(C_{\mathrm{a}}^{j}+C_{\mathrm{g}}^{j}\right) C_{\mathrm{b}}^{j} / C_{\Sigma}^{j}$.

We consider an oscillator with frequency much higher than all qubit frequencies, $\hbar / \sqrt{L C_{s}} \gg E_{C}^{j}, E_{\mathrm{a}}^{j}, E_{\mathrm{b}}^{j}$, and further assume $\sqrt{L / C_{s}} \ll \hbar / e^{2}$. This ensures that both the average value and the fluctuations of the oscillator flux $\Phi_{L}$ are much smaller than $\Phi_{0}$, and the oscillator can be adiabatically eliminated, cf. Ref. 2. Below, we further discuss the condition of adiabaticity. In the process we transform the qubit phase as $\phi^{j} \rightarrow \tilde{\phi}^{j}=\phi^{j}-k_{\mathrm{a}}^{j} \phi_{L}$. We further consider the charge regime $E_{C}^{j}=e^{2} /\left(2 C_{\Sigma}^{j}\right) \gg$ $E_{\mathrm{a}}^{j}, E_{\mathrm{b}}^{j}$. If for each qubit the gate voltage is chosen close to a charge degeneracy point, we can employ a two-state approximation with relevant charge states denoted as $|n=1\rangle$ and $|n=0\rangle$. After a transformation $\tilde{\phi}^{j} \rightarrow \theta^{j}=\tilde{\phi}^{j}-\phi_{\mathrm{x}}^{j} / 2$ and the gauge choice $\cos \theta^{j} \rightarrow \sigma_{x}^{j} / 2$ and $\sin \theta^{j} \rightarrow \sigma_{y}^{j} / 2$, we find the effective Hamiltonian

$$
H_{\mathrm{eff}}=\sum_{j=1}^{N} H_{\text {single }}^{j}-\frac{L}{2}\left(\sum_{j=1}^{N} I^{j}\right)^{2} .
$$

The single-qubit Hamiltonian of qubit $j$ and its contribution to the current through the inductor are given by

$$
\begin{aligned}
H_{\text {single }}^{j} & =-\frac{1}{2}\left(B_{x}^{j} \sigma_{x}^{j}+B_{y}^{j} \sigma_{y}^{j}+B_{z}^{j} \sigma_{z}^{j}\right), \\
I^{j} & =\frac{1}{2}\left(I_{x}^{j} \sigma_{x}^{j}+I_{y}^{j} \sigma_{y}^{j}\right),
\end{aligned}
$$

where

$$
\begin{aligned}
& B_{x}=\left(E_{\mathrm{a}}+E_{\mathrm{b}}\right) \cos \left(\phi_{\mathrm{x}} / 2\right), \\
& B_{y}=\left(-E_{\mathrm{a}}+E_{\mathrm{b}}\right) \sin \left(\phi_{\mathrm{x}} / 2\right), \\
& B_{z}=4 E_{C}(q / e-1) .
\end{aligned}
$$

In Eqs. (44) (and below, where it does not lead to confusion) we omit the qubit index $j$. The current of each qubit is

$$
\begin{aligned}
& I_{x}=\left[k_{\mathrm{a}} \cdot I_{\mathrm{a}}+\left(1-k_{\mathrm{a}}\right) \cdot I_{\mathrm{b}}\right] \sin \left(\phi_{\mathrm{x}} / 2\right) \\
& I_{y}=\left[k_{\mathrm{a}} \cdot I_{\mathrm{a}}+\left(k_{\mathrm{a}}-1\right) \cdot I_{\mathrm{b}}\right] \cos \left(\phi_{\mathrm{x}} / 2\right),
\end{aligned}
$$

where the critical currents of the junctions above and below the island of a qubit are denoted by $I_{\mathrm{a} / \mathrm{b}}=$ $2 \pi E_{\mathrm{a} / \mathrm{b}} / \Phi_{0}$.

The design of Ref. 15 with longitudinal coupling is recovered for symmetric Josephson energies and capacitances $\left(E_{\mathrm{a}}=E_{\mathrm{b}}\right.$ and $\left.k_{\mathrm{a}}=1 / 2\right)$. On the other hand, the coupling is always transverse $\frac{19}{19}$ for $E_{\mathrm{a}}=0$ or $E_{\mathrm{b}}=0$.

Conditions for longitudinal coupling. We will determine now under which conditions the coupling is longitudinal, that is, the single-qubit terms commute with the coupling term,

$$
\left[H_{\text {single }}^{j}, L \sum_{k, l} I^{k} I^{l}\right]=0 \text { for all } j .
$$

First, we assume operation at the charge degeneracy point, $B_{z}^{j}=0$, of each qubit $j$. Here decoherence is slowest, and only the tunneling energy part, $U^{j}=$ $-(1 / 2) B_{x}^{j} \sigma_{x}^{j}-(1 / 2) B_{y}^{j} \sigma_{y}^{j}$, of the single-qubit energy remains. Since the current operators of different qubits commute, the condition (6) is satisfied if and only if $\left[I^{j}, U^{j}\right]=0$ for each qubit $j$, and it is sufficient to consider each qubit separately. This commutator vanishes when the following condition is fulfilled for each qubit

$$
k_{\mathrm{a}} E_{\mathrm{a}}^{2}+\left(k_{\mathrm{a}}-1\right) E_{\mathrm{b}}^{2}+\left(2 k_{\mathrm{a}}-1\right) E_{\mathrm{a}} E_{\mathrm{b}} \cos \phi_{\mathrm{x}}=0 .
$$


As expected, this condition of longitudinal coupling is $2 \pi$-periodic in the applied phases. Apart from trivial cases, where either single-qubit and coupling terms vanish $\left(E_{\mathrm{a}}=E_{\mathrm{b}}=0\right)$, or where the connection on one side of the island is broken $\left(E_{\mathrm{a}}=0\right.$ and $k_{\mathrm{a}}=1$ or $E_{\mathrm{b}}=0$ and $k_{\mathrm{a}}=0$ ), this condition can only be fulfilled in the following two cases: (i) $E_{\mathrm{a}}=E_{\mathrm{b}}$ and $k_{\mathrm{a}}=1 / 2$, or (ii)

$$
\cos \phi_{\mathrm{x}}=\frac{\kappa-\epsilon^{2}}{(1-\kappa) \epsilon}
$$

Here we introduced the ratios of Josephson couplings and of capacitances above and below the junction,

$$
\epsilon \equiv \frac{E_{\mathrm{a}}}{E_{\mathrm{b}}} \quad, \quad \kappa \equiv \frac{1-k_{\mathrm{a}}}{k_{\mathrm{a}}}=\frac{C_{\mathrm{a}}+C_{\mathrm{g}}}{C_{\mathrm{b}}} .
$$

Note that case (i) corresponds to the symmetric situation: equal Josephson couplings and capacitances on both sides of the island $(\epsilon=\kappa=1)$. While the former may be adjusted by replacing junctions by SQUID loops, the spread in the values of the capacitances can not be avoided in real experiments.

Tuning to longitudinal coupling. We will now show that for case (ii) for a wide range of design parameters in spite of some spread - one can tune to longitudinal coupling by proper choice of the applied flux for each qubit. Especially, condition (8) can be satisfied even when capacitances and Josephson energies of the fabricated setup deviate from their nominal values. Via the applied fluxes in the design of Fig. 1, the phases $\phi_{\mathrm{x}}$ can be tuned in the full range between 0 and $2 \pi$, and the condition (8) can be satisfied, when

$$
\text { 1) } \kappa \leq \epsilon \leq 1 \quad \text { or } \quad 2) 1 \leq \epsilon \leq \kappa
$$

This parameter range, where it is possible to tune to longitudinal coupling, is shown in Fig. 2 as region I (green). On the other hand, in region II of this diagram, purely longitudinal coupling can not be achieved.

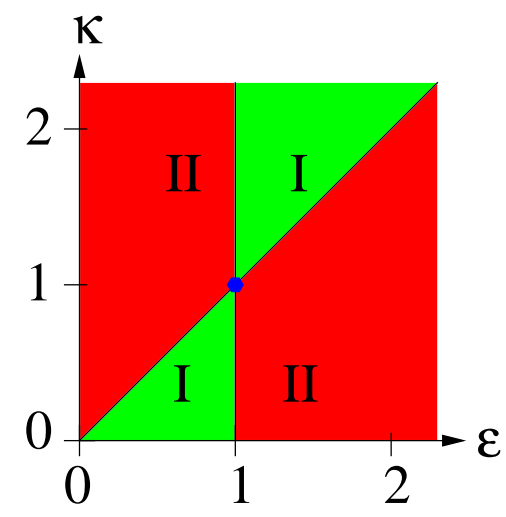

FIG. 2: (Color online) Stability diagram for a Josephson charge qubit. In region I tuning to longitudinal coupling by applied magnetic fluxes is possible. In region II the coupling always has a transverse component.
Only for the symmetric case, $\kappa=\epsilon=1$, which corresponds to a single point in the diagram shown in Fig. 2, the coupling is longitudinal for an arbitrary value of the parameter $\phi_{\mathrm{x}}$. For this reason, the symmetric design appears most appealing. However, it is unstable in the sense that small deviations of the parameters $\epsilon$ or $\kappa$ from the nominal value of 1 may bring the system into region II, where longitudinal coupling cannot be achieved. Therefore, in order to be able to tune to stable longitudinal coupling, one should aim for design parameters inside region I of the phase diagram. Since the commutation relation should be satisfied for all qubits, one needs to build each qubit with parameters in the proper range. Further, one needs to have for each qubit an individual control parameter $\phi_{\mathrm{x}}$ (as opposed to a common flux through the inductor used in Ref. 15).

Stability of longitudinal coupling with respect to fluctuations in the control fluxes. Time-dependent fluctuations or drifts in the applied fluxes will shift the operation point from the desired values given by Eq. (8). We want to estimate for which values of the design parameters these deviations lead to the weakest instability of the longitudinal coupling. For this purpose we introduce a measure for the residual transverse-coupling strength. If one regards the single qubit terms at the degeneracy point and the current of each qubit as vectors, $\left(B_{x}, B_{y}\right)$ and $\left(I_{x}, I_{y}\right)$, in the $x-y$ plane, the commutation between both corresponds to parallel vectors. On the other hand, the $z$-component of their cross product, $B_{x} I_{y}-B_{y} I_{x}$, which is also proportional to the lhs. of Eq. (7), is a measure for the contribution of one qubit to the transverse coupling. To gain independence of the total coupling strength, we consider the ratio of transverse and longitudinal coupling strengths,

$$
t=\frac{B_{x} I_{y}-B_{y} I_{x}}{B_{x} I_{x}+B_{y} I_{y}} .
$$

The numerator and denominator vanish for purely longitudinal or transverse coupling, respectively. Larger values of $|t|$ correspond to stronger transverse coupling. We find

$$
t=\frac{k_{\mathrm{a}} E_{\mathrm{a}}^{2}+\left(k_{\mathrm{a}}-1\right) E_{\mathrm{b}}^{2}+\left(2 k_{\mathrm{a}}-1\right) E_{\mathrm{a}} E_{\mathrm{b}} \cos \phi_{\mathrm{x}}}{E_{\mathrm{a}} E_{\mathrm{b}} \sin \phi_{\mathrm{x}}} .
$$

When $\phi_{\mathrm{x}}=\phi_{\mathrm{x} 0}+\delta \phi_{\mathrm{x}}$ deviates from the value $\phi_{\mathrm{x} 0}$ satisfying the condition (8), the variation of the relative coupling strength in the linear order is governed by

$$
\left.\frac{\partial t}{\partial\left(\delta \phi_{\mathrm{x}}\right)}\right|_{\delta \phi_{\mathrm{x}}=0}=1-2 k_{\mathrm{a}}=\frac{\kappa-1}{\kappa+1} .
$$

Note that this expression holds in region I of the stability diagram for the special values of the flux bias (8), at which $t$ vanishes. Thus, on one hand, to tune to longitudinal coupling one has to aim inside region I, not too close to its boundary; on the other hand, in order to minimize the sensitivity to fluctuations (13), one should keep the value of $\kappa$ close to 1 . 
Slow and fast oscillators. So far we considered a fast oscillator, $\hbar \omega_{L C}=\hbar / \sqrt{L C_{s}} \gg E_{\mathrm{qb}} \sim E_{C}^{j}, E_{\mathrm{a}}^{j}, E_{\mathrm{b}}^{j}$, which could be adiabatically eliminated. Let us briefly discuss the opposite case of a slow oscillator (cf. Ref. 20; note that $\omega_{L C}$ decreases with the number $N$ of qubits). For this purpose, it is convenient to split the operators $I^{j}$ into longitudinal and transverse parts, $I^{j}=I_{\text {long }}^{j}+I_{\text {trans }}^{j}$. Since $I_{\text {long }}^{j}$ is a slow variable (as long as the single-qubit Hamiltonian is kept fixed), the longitudinal coupling term is of the same form as above, $-\frac{L}{2}\left(\sum_{j=1}^{N} I_{\text {long }}^{j}\right)^{2}$. Notably, the transverse coupling is suppressed: $I_{\text {trans }}$ varies at frequencies $\sim E_{\mathrm{qb}} \gg \omega_{L C}$, at which the response of the oscillator is weak, and this results in a transverse inductive coupling suppressed by a factor $\left(\omega_{L C} / E_{\mathrm{qb}}\right)^{2}$ relative to the longitudinal coupling. Thus, in case of a slow oscillator the longitudinal coupling is even more stable. This description of the coupling applies on time scales longer than $\omega_{L C}^{-1}$. This constraint does not allow a decrease in $\omega_{L C}$ indefinitely and, in particular, limits the scalability, i.e., the number $N$ of qubits.

Comments. (i) Besides working in the parameter regime given in Eq. (10), one should keep the gate capacitances $C_{\mathrm{g}}$ small, in order to decouple the qubits from the electromagnetic environment. These can be conflicting requirements, since the ratio of Josephson energies $E_{\mathrm{a}} / E_{\mathrm{b}}$ is usually close to that of the capacitances $C_{\mathrm{a}} / C_{\mathrm{b}}$ in a fabricated sample. This problem can be lifted by employing an additional capacitance in parallel to either of the qubit junctions.

(ii) We assumed individually controllable fluxes in qubit loops. In experiment, fluxes are controlled via multiple current lines. However, cross couplings may appear, which can be overcome ${ }^{9}$.

(iii) For the design as discussed so far, there is only one control flux for each qubit which is used to tune to the point of longitudinal coupling. Since one would like to control the coupling strength independently, additional control parameters are desirable. One possibility is to replace simple junctions by SQUID loops with additional control fluxes. These can be used to change the effective Josephson energies and therefore the coupling strength. Furthermore, when one keeps the ratio of effective Josephson energies constant, one can stay at the point of longitudinal coupling, while changing the longitudinal coupling strength. We emphasize the difference to the proposal of Ref. 15, where only one common flux in "outer loops" is applied, and longitudinal coupling can not be achieved in this way for asymmetric design parameters.

(iv) Alternatively, tunable coupling strength may be achieved by a standard replacement of the inductor with a Josephson junction in the phase regime, see, e.g., Ref.22. The tunability can be achieved by current-biasing this junction $\frac{17}{2}$. This has an additional advantage since in an experiment currents can be switched faster than fluxes. In order to produce longitudinal coupling in such a situation, the condition of Eq. (8) needs to be slightly modified; one has to add the additional phase applied by the control current to $\phi_{\mathrm{x}}$ for each qubit.

(v) Searching for longitudinal coupling which is stable against weak flux fluctuations, one may consider more involved designs, in particular, with Josephson junctions replaced by dc-SQUID loops or even more junctions in parallel. This would provide more tuning parameters (magnetic fluxes in the loops) and potentially the possibility to find a better operation point. However, such a point should be stable against fluctuations in all control fluxes. Moreover, the additional fluxes will also fluctuate and increase the noise level. One can show that it is impossible to stabilize the longitudinal coupling in this manner 21 .

In summary, we analyzed, for a system of inductively coupled charge qubits, the conditions for longitudinal inter-qubit coupling, i.e., a coupling that commutes with single-qubit terms. Earlier suggestions relied on precise symmetries of parameters of various junctions. We studied the stability of the longitudinal coupling with respect to deviations from this nominal symmetry, which is unavoidable during fabrication. We have shown that in a simple design one can reach longitudinal coupling by keeping the nominal parameters in a certain range, away from the symmetry point. For such a circuit, one can always compensate for deviations of the parameters of the fabricated circuit by tuning control fluxes. Fluctuations of magnetic fluxes render the longitudinal character unstable for any circuit of the considered type. We found the conditions when this instability is weak.

This work is part of the EU IST project EuroSQIP, and was also supported by the RSSF (YM) and by Graduiertenkolleg "Kollektive Phänomene im Festkörper" (CH). We acknowledge valuable discussions with M. Wallquist.
1 M.A. Nielsen and I.L. Chuang, Quantum Computation and Quantum Information, Cambridge University Press (2000).

2 Yu. Makhlin, G. Schön, and A. Shnirman, Rev. Mod. Phys. 73, 357 (2001).

3 M. H. Devoret and J. M. Martinis, Superconducting qubits, in Quantum entanglement and information processing, page 443, edited by D. Estève, J. M. Raimond, and J. Dalibard (Elsevier, 2003).

${ }^{4}$ G. Wendin and V. S. Shumeiko, in Handbook of Theoretical and Computational Nanotechnology, Vol. 3, page 223, edited by M. Rieth and W. Schommers (ASP, 2006).

${ }^{5}$ Yu. A. Pashkin, T. Yamamoto, O. Astafiev, Y. Nakamura, D. V. Averin and J. S. Tsai, Nature (London) 421, 823 (2003).

6 T. Yamamoto, Yu. A. Pashkin, O. Astafiev, Y. Nakamura 
and J. S. Tsai, Nature (London) 425, 941 (2003).

7 A. Izmalkov, M. Grajcar, E. Il'ichev, Th. Wagner, H.-G. Meyer, A. Yu. Smirnov, M. H. S. Amin, A. Maassen van den Brink and A. M. Zagoskin, Phys. Rev. Lett. 93, 037003 (2004).

8 J. B. Majer, F. G. Paauw, A. C. J. ter Haar, C. J. P. M. Harmans and J. E. Mooij, Phys. Rev. Lett. 94, 090501 (2005).

9 R. McDermott, R. W. Simmonds, M. Steffen, K. B. Cooper, K. Cicak, K. D. Osborn, S. Oh, D. P. Pappas and J. M. Martinis, Science 307, 1299 (2005).

10 A. Wallraff, D. I. Schuster, A. Blais, L. Frunzio, R.S. Huang, J. Majer, S. M. Girvin and R. J. Schoelkopf, Nature (London) 431, 162 (2004).

11 I. Chiorescu, P. Bertet, K. Semba, Y. Nakamura, C. J. P. M. Harmans and J. E. Mooij, Nature (London) 431, 159 (2004).

12 S. H. W. van der Ploeg, A. Izmalkov, A. Maassen van den Brink, U. Hübner, M. Grajcar, E. Il'ichev, H.-G. Meyer and A. M. Zagoskin, Phys. Rev.
Lett. 98, 057004 (2007).

13 T. Hime, P. A. Reichardt, B. L. T. Plourde, T. L. Robertson, C. E. Wu, A. V. Ustinov and J. Clarke, Science 314, 1427 (2006).

14 Yu. Makhlin, G. Schön, and A. Shnirman, Nature (London) 398, 305 (1999).

15 J. Q. You, J. S. Tsai, and F. Nori, Phys. Rev. Lett. 89, 197902 (2002), cond-mat/0306208.

16 J. Q. You, Chi-Hang Lam, and H. Z. Zheng, Phys. Rev. B 63, 180501(R) (2001).

17 J. Lantz, M. Wallquist, V. S. Shumeiko and G. Wendin, Phys. Rev. B 70, 140507(R) (2004).

18 M. Wallquist, J. Lantz, V. S. Shumeiko and G. Wendin, New J. Phys. 7, 178 (2005).

19 A. Shnirman, G. Schön, and Z. Hermon, Phys. Rev. Lett. 79, 2371 (1997).

20 A. Sørensen and K. Mølmer, Phys. Rev. A 62, 022311 (2000).

21 C. Hutter, PhD thesis, University of Karlsruhe (2007). 